\title{
Multiwall carbon nanotube-filled natural rubber: Electrical and mechanical properties
}

\author{
L. Bokobza* \\ Laboratoire PPMD, ESPCI ParisTech, 10 rue Vauquelin, 75231 Paris Cedex, France
}

Received 11 July 2011; accepted in revised form 25 September 2011

\begin{abstract}
The influence of multiwall carbon nanotube (MWNTs) contents on electrical and mechanical properties of MWNTs-reinforced natural rubber (NR) composites is studied. The volume resistivity of the composites decreases with increasing the MWNTs content and the electrical percolation threshold is reached at less than $1 \mathrm{phr}$ of MWNTs $(\mathrm{phr}=\mathrm{parts}$ of filler by weight per hundred parts of rubber). This is caused by the formation of conductive chains in the composites. Electrical measurements under uniaxial deformation of a composite carried out at a filler loading above the percolation threshold, indicate a gradual disconnection of the conducting network with the bulk deformation. The drop in the storage modulus $G^{\prime}$ with the shear strain amplitude (Payne effect) is also attributed to a breakdown of the filler network. Considerable improvement in the stiffness is obtained upon incorporation of MWNTs in the polymer matrix but the main factor for reinforcement of NR by MWNTs appears to be their high aspect ratio rather than strong interfacial interaction with rubber. The tensile strength and the elongation at break of the composites are reduced with regard to the unfilled sample. This is probably due to the presence of some agglomerates that increase with the nanotube content. This hypothesis is confirmed by a cyclic loading of the composites where it is seen that the deformation at break occurs at a much higher level of strain in the second stretch than in the first one. The overall significant property improvements are the result of a better nanotube dispersion attributed to the combined use of tip sonication and cyclohexane as dispersion aids during composite processing.
\end{abstract}

Keywords: polymer composites, rubber, reinforcements, carbon nanotubes

\section{Introduction}

Polymer nanocomposites have attracted a huge scientific interest because they allow the design of high-performance materials that exhibit significant improved properties with regard to the pristine polymer. The extent of improvement generally depends on several parameters including the size of the particles, their aspect ratio, their state of dispersion and their surface chemical characteristics that determine the interaction between the filler and the polymer chains and thus the interface of the polymer-filler system [1,2].

Polymer-carbon nanotube composites have attracted particular interest because the structural characteristics of carbon nanotubes such as their high aspect ratio, high surface area available for stress transfer as well as their exceptionally high Young's modulus and excellent electrical and thermal properties, are expected to allow the emergence of a new generation of ultra-lightweight and extremely strong composite materials [3-5]. In fact, one of the biggest challenges is to obtain a homogeneous dispersion of CNTs in a polymer matrix because van der Waals interactions between individual tubes lead to significant aggregation and agglomeration that reduce the expected property improvements. A homogeneous distribution and a good dispersion are essential for mechanical reinforcement of polymers. In the case of anisotropic electrical conductive inclusions, a good dispersion allows the formation of an inter-

\footnotetext{
${ }^{*}$ Corresponding author, e-mail: Liliane.Bokobza@espci.fr (c) BME-PT
} 
connecting filler network at a low filler content characterized by a sharp drop, by several orders of magnitude, in the electrical resistivity of the composites reaching the so-called percolation threshold. In fact, it is one of the major attributes of carbon nanotubes to provide electrical conduction at a very low filler loading, this property being required in commercial applications to dissipate electrostatic charge.

This paper reports investigations carried out on natural rubber filled with multiwall carbon nanotubes. The state of nanotube dispersion is evaluated from transmission electron microscopy and electrical properties in the isotropic and uniaxially stretched states of the composite are examined in addition to mechanical measurements.

\section{Experimental part}

\subsection{Materials}

Multiwall carbon nanotubes (MWNTs) were purchased from Nanocyl S.A. (Belgium). In this study, we have used the Nanocyl 7000 series (purity: $90 \%$ ) produced via the catalytic carbon vapor deposition process without any further purification. Their average diameter and length are around $10 \mathrm{~nm}$ and $1.5 \mu \mathrm{m}$ respectively and their surface area between 250 and $300 \mathrm{~m}^{2} \cdot \mathrm{g}^{-1}$.

Non-vulcanized natural rubber, containing all the vulcanization ingredients was provided by Formix (Orléans, France). It was compounded with sulfur (1.5 phr), zinc oxide ( $3 \mathrm{phr})$, stearic acid ( $2 \mathrm{phr}$ ), cyclohexylbenzothiazole sulfenamide (1.5 phr), $(\mathrm{phr}=$ parts by weight per hundred parts of rubber).

\subsection{Composite processing}

One of the main factors that have a large influence on the physical performance of a filled composite is the state of filler dispersion within the host polymer matrix. Carbon nanotubes can easily form bundles and this aggregation decreases their aspect ratio and reduces their efficiency as reinforcing agents for high strength polymer composites. Different processing methods, including in-situ polymerization, solution mixing, surfactant-assisted processing and melt compounding, have been used to optimize the dispersion of CNTs in the polymeric medium in order to fully exploit the potential of these materials.

In this work, solution mixing which is one of the most common methods, has been used for fabricat- ing MWNTs/NR composites. The general protocol is to mix both components in a suitable solvent and evaporate the latter before proceeding to the crosslinking process and film formation. The most efficient dispersion of the nanotubes in a solvent is achieved by tip sonication with sonication conditions determined in such a way not to degrade the nanotubes.

An appropriate amount of MWNTs was dispersed into cyclohexane $(0.1 \mathrm{mg} / \mathrm{ml})$ by sonicating the suspension for $30 \mathrm{~min}$ ( 3 times separated by a rest of $30 \mathrm{~min}$ ) using a Vibra-Cell VCX 500 operating at $40 \%$ amplitude with on and off cycles respectively equal to 4 and 2 seconds. Cyclohexane was used to disperse the nanotubes because it can also dissolve natural rubber and is less dangerous than toluene well known to be is a good solvent for rubbers.

The gum containing the rubber and all the ingredients of formulation was mixed separately in cyclohexane under magnetic stirring until complete dissolution then mixed with the MWNTs dispersion. The mix of polymer and MWNTs dispersions is stirred until evaporation of the major part of cyclohexane then put under vacuum at $50^{\circ} \mathrm{C}$ for one day for total removal of remaining solvent.

The unfilled and filled samples were then cured into plaques at $170^{\circ} \mathrm{C}$ during $20 \mathrm{~min}$ under a pressure of 120 bar in a standard hot press.

\subsection{Characterization techniques}

The state of dispersion of the filler particles was examined by transmission electron microscopy (TEM) by means of a JEOL JEM-2010 Electron Microscope, operating at $200 \mathrm{kV}$. Ultrathin sections (50-60 nm) were cut at $-90^{\circ} \mathrm{C}$ by using an Ultracut $\mathrm{S}$ ultramicrotome from Leica fitted with a diamond knife from Diatome.

Electrical resistivity measurements were determined on samples of $10 \times 20 \times 0.2 \mathrm{~mm}^{3}$ by measuring their resistance on a high resistance meter (Keithley 6517A) between two conductive rubber electrodes with an alternating voltage of $1 \mathrm{~V}$. This alternating voltage is needed to avoid a background current effect. The measured resistances $R$ were then converted into volume resistivity $\rho$ by using Equation (1):

$\rho=\frac{R S}{d}$ 
where $S$ is the cross-sectional area perpendicular to the current and $d$ the thickness of the sample between the two electrodes.

The strain dependence of electrical resistivity was carried out on strips (size: $40 \mathrm{~mm} \times 6 \mathrm{~mm} \times 0.3 \mathrm{~mm}$ ) stretched with a manual stretching machine. The film under study is clamped between the jaws of the stretching machine and two copper plates attached to the jaws of the machine are connected to the high-resistance meter.

Under the assumption that the volume remains constant during deformation, the resistivity was obtained from the measured resistance, $R$, from Equation (2):

$$
\rho=\frac{R S_{0}}{\alpha^{2} L_{0}}
$$

where $S_{0}$ and $L_{0}$ are respectively, the initial crosssectional area and length between the two clamps and $\alpha$ is the extension ratio, which is the ratio of the length of the sample in the direction of strain to the initial length before deformation.

The dynamic properties of the vulcanizates were measured by means of a Anton Paar Rheometer at

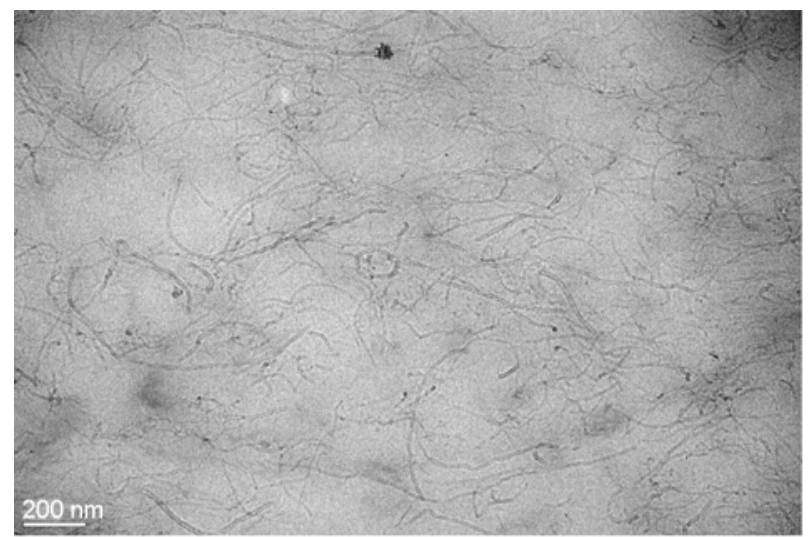

a)

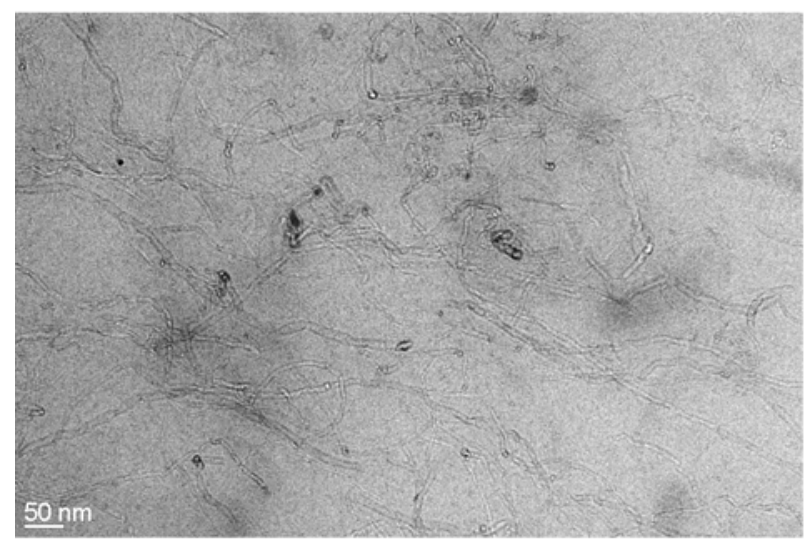

c)
$1 \mathrm{~Hz}$ sinusoidal oscillation, using disc specimens with thicknesses of $2 \mathrm{~mm}$, diameters of $8 \mathrm{~mm}$, operated in a shear mode.

The tensile tests were performed at room temperature on a standard tensile Instron machine, model 5565 equipped with a $10 \mathrm{~N}$ load cell and a video extensometer. The strips (length around $20 \mathrm{~mm}$ between the jaws, width around $5 \mathrm{~mm}$ and thickness between 200 and $300 \mu \mathrm{m}$ ) were marked with two dots with a white marker for their recognition by the video extensometer then stretched at a strain rate of $0.1 \mathrm{~s}^{-1}$.

\section{Results and discussion \\ 3.1. Transmission electron microscopy analysis (TEM)}

The state of dispersion, which significantly affects the electrical and mechanical properties of the material, can be evidenced by TEM analysis of the composites.

TEM images, taken at different scales for the NR composite filled with $3 \mathrm{phr}$ of MWNTs, are seen in Figure 1. Figures $1 \mathrm{a}-\mathrm{c}$ show a rather homogeneous

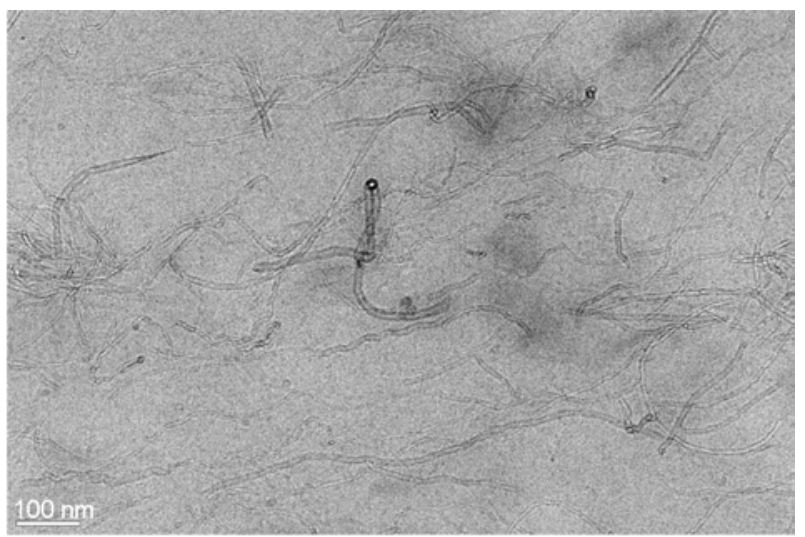

b)

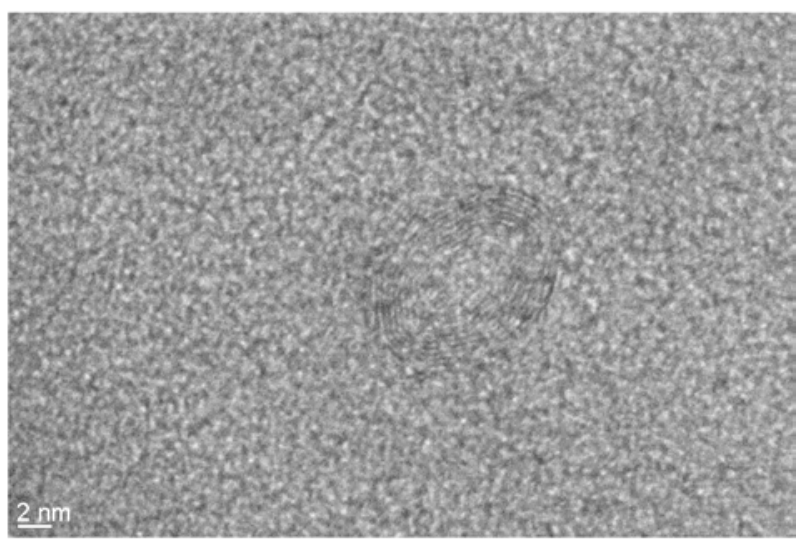

d)

Figure 1. TEM images of NR filled with 3 phr of MWNTs taken at different (a, b, c) scales. d) shows the graphene layers of an individual tube 
distribution of the nanotubes in the elastomeric matrix and in Figure 1d are magnified the graphene shells with the hollow core of an individual tube.

\subsection{Electrical properties}

Carbon nanotubes have largely demonstrated their ability to provide electrical conduction when incorporated into polymeric media usually considered as electrical insulators. From a great number of results in the literature, it is now evident that very small quantities of carbon nanotubes are required to get relatively high values of electrical conductivity and this property is expected to open the way for a wide range of industrial applications.

The electrical conduction process depends on several parameters, mainly on filler concentration. But processing techniques intended to improve dispersion of CNTs in the host matrix also influence the electrical conductivity of the final material. On the other hand, factors such as nanotube type, polymer matrix, filler-matrix interactions and filler orientation are key factors in determining the electrical properties. At a given amount of conductive particles (carbon black or carbon nanotubes), called the percolation threshold, a continuous network of filler is formed across the matrix and the material undergoes a sudden transition from an insulator to a conductor. For composites containing conventional conducting fillers such as carbon black (CB), carbon nanofibers or graphite, depending on the structural properties of the particles, the percolation threshold is achieved for a filler content as high as $10-50 \mathrm{wt} \%$, which may result in a composite with poor mechanical properties and high density. Thongruang et al. [6] showed that the percolation-threshold concentration in composites is around $10-15 \mathrm{wt} \%$ for carbon fiber and high structure carbon black and around $40-50 \mathrm{wt} \%$ for low structure carbon black and graphite. Carbon nanotubes yield adequate conductivity at a much lower filler content on account of their high aspect ratios thus retaining the desired mechanical properties of the resulting material. Another way to enhance electrical conductivity has been shown to incorporate simultaneously hybrid fillers of carbon nanotubes and carbon black [7, 8]. It was demonstrated that when $\mathrm{CB}$ particles are added into the nanocomposites containing CNTs, the result is a better overall dispersion due to synergistic effects arising between the two different fillers and the formation of connected structures by bridging uncontacted particles. But, the replacement of carbon black by carbon nanotubes alone for the synthesis of electrically conducting polymer composites is very promising for the design of lightweight materials for numerous future applications.

As seen in Figure 2 which shows the effect of filler loading on the volume resistivity of MWNTs-filled composites based on insulating natural rubber, the decrease in resistivity is observed from 0.5 to $1 \mathrm{phr}$. According to Mamunya et al. [9], at the beginning of the transition region, an infinite conductive cluster is formed and, consequently, the composite becomes conductive. The higher aspect ratio of the nanotube bundles which increases the probability of particleparticle contacts explain the low percolation threshold. With regard to previous reported results [10], thanks to improved dispersion methods, the percolation threshold is shifted to a lower nanotube content and from this point of view, measurements of electrical resistivity appears as an indirect tool to evaluate the state of dispersion. As mentioned above, an important factor that controls the performance and especially the electrical properties of CNTsreinforced composites is the state of dispersion of CNTs.

Because the resistivity is very sensitive to any change in filler distribution, electrical properties of composite filled with conductive particles should be affected by a mechanical deformation. Significant changes in electrical conductivity against degree of elongational strain have already been reported in the literature [11-15].

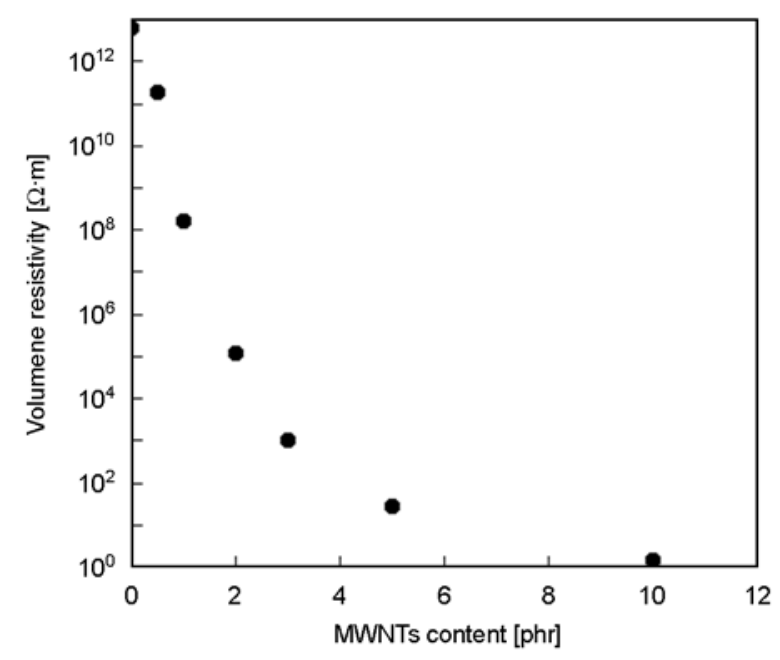

Figure 2. Volume resistivity against nanotube loading for NR composites 
Typical strain dependences of volume resistivity are shown in Figure 3: the results are related to a NR sample filled with $3 \mathrm{phr}$ of MWNTs that is at a filler content above the percolation threshold (determined around $0.5 \mathrm{phr}$ of filler). Tests are performed as follows:

- 1 : stretching to $\varepsilon=100 \%$ then release,

-2 and 3 : second stretching to $\varepsilon=200 \%$ then release,

-4 and 5: third stretching until failure.

A gradual increase in resistivity is obtained in the first step as result of a breakdown of contacts and increasing distances between the conducting inclusions. After total unloading of the sample (step 2), the resistivity is much higher than that observed for the undeformed material thus showing that the contacts are not reformed after removal of the stress. A second stretching leads to a decrease in resistivity until the point where the first and second stretching meet. At this point, we can consider that the sample is in the same state as it was at the first stretching. It is interesting to point out that this part of the curve (unloading + second stretch) is completely reversible as shown in the paper of Ciselli et al. [16] related to composites based on ethylene-propylene-dienemonomer (EPDM) filled with MWNTs. After the point where the first and second stretchings meet, the curve join up the first stretching curve till $\varepsilon=$ $200 \%$ (step 3) and the resistivity increases again due to the breakdown of new contacts that were not affected during the first stretching. A further removal of the stress leads to a higher value of resistivity than that obtained in the first unloading cycle and

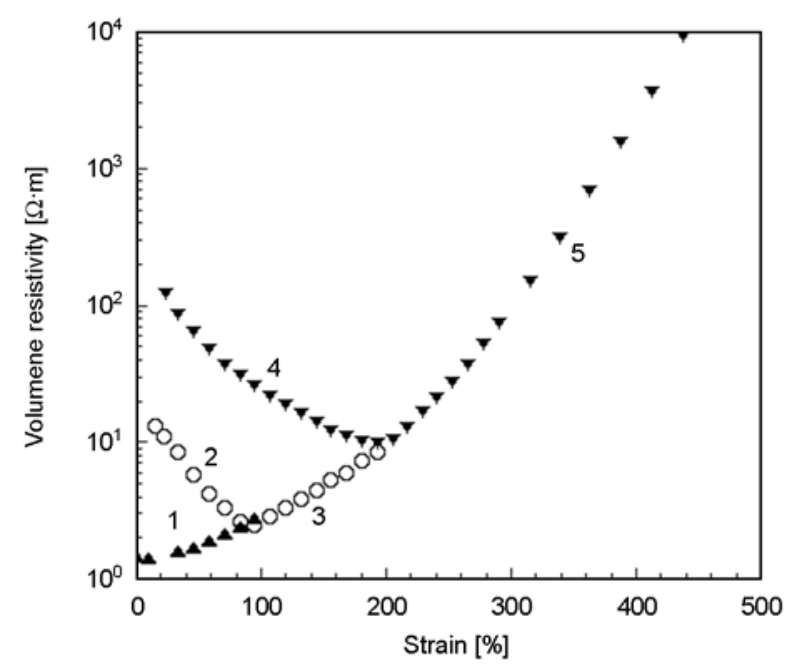

Figure 3. Strain dependence of the electrical resistivity for NR filled with 3 phr of MWNTs the third stretching displays a decreease (step 4) then increase (step 5). The strain dependence of electrical resistivity described here for NR composites is quite similar to that obtained in other rubbery matrices like SBR [17] or EPDM [18].

\subsection{Mechanical properties}

\subsubsection{Low-strain dynamic behavior}

Filled elastomers have the specific ability to dissipate an important part of mechanical energy during deformation. At small strains (typically for shear strains below $100 \%$ ), those materials exhibit a nonlinear viscoelastic behavior, known as the 'Payne effect' and characterized by a drop in the elastic modulus $G^{\prime}$ when the shear strain amplitude increases. The Payne effect in composites filled with conventional fillers (carbon black or silica) has been the subject of numerous studies on both experimental and theoretical aspects [19-22] but its origin is still controversial. The most commonly accepted picture is the breakdown of a filler network formed by filler-filler interactions. The percolated network is progressively broken by increasing the strain amplitude.

Figure 4 displays the strain dependence of the storage modulus of the unfilled NR and of MWNTsfilled composites. While the unfilled elastomer does not display any change in the storage modulus with strain amplitude, at least in the investigated strain domain, the observed behaviors for filled compounds are similar to what was mentioned in the lit-

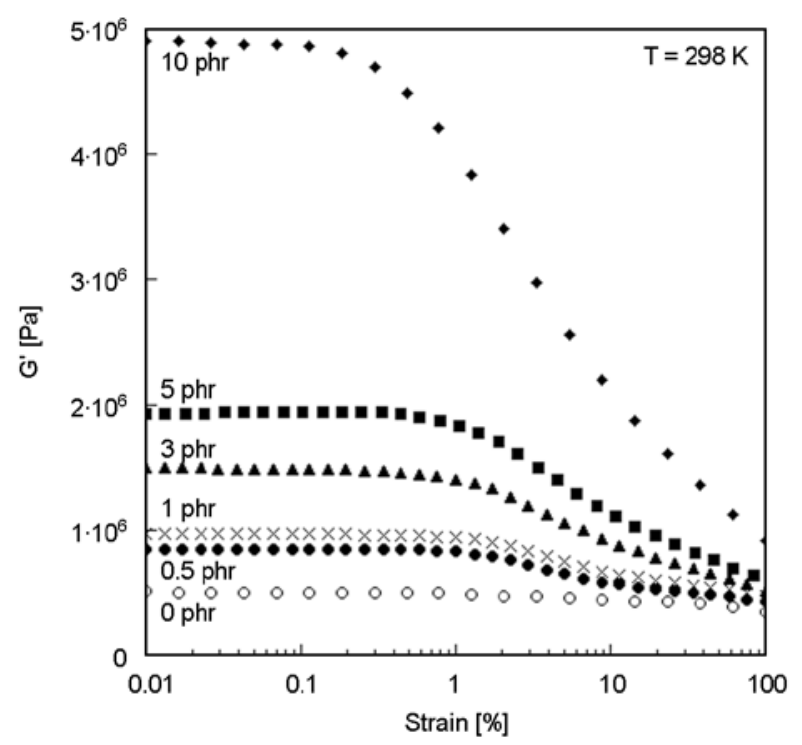

Figure 4. Strain dependence of the storage modulus of unfilled NR and of MWNTs/NR composites at room temperature 


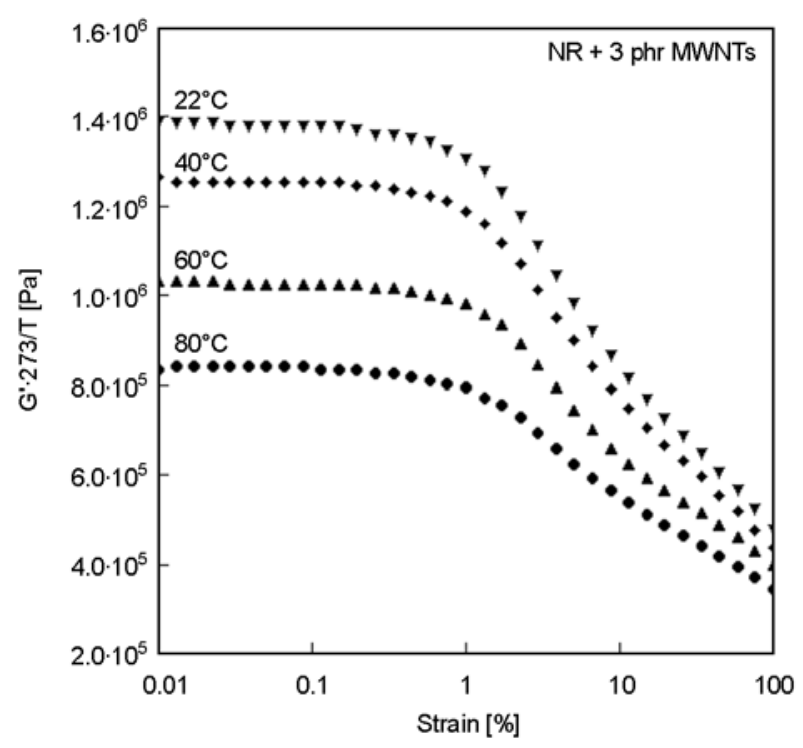

Figure 5. Temperature dependence of the storage modulus for NR filled with 3 phr of MWNTs

erature for carbon-black filled rubbers, that is an increase in amplitude of the Payne effect with the amount of reinforcing particles, because the linear part of the storage modulus typically below $0.1 \%$ strain strongly increases with the filler loading. But as shown in Figure 4, a Payne effect is observed till $0.5 \mathrm{phr}$ while much larger amounts of carbon black are required to give rise to a storage modulus drop with the strain amplitude [23].

In the case of an unfilled rubber network, the modulus should increase proportionally with temperature because of its entropic nature. For all the filled systems, the modulus values should then be corrected by the entropic factor $273 / \mathrm{T}$, in order to get rid of the modulus dependence of the rubber part

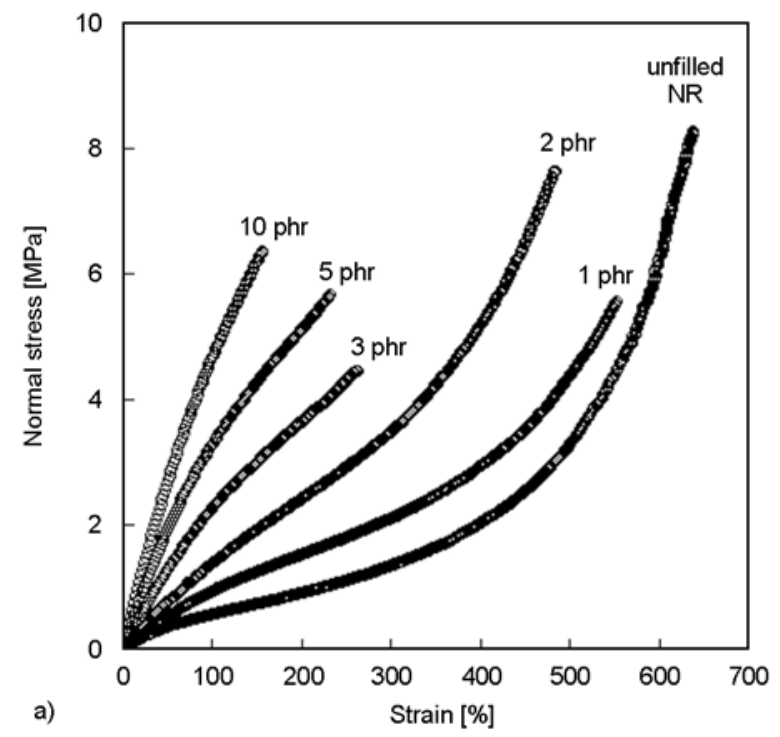

due to temperature [20]. As a typical example, the temperature dependence of the storage modulus is shown in Figure 5 for the $3 \mathrm{phr}$ MWNTs/NR composite. As already observed in conventional composites, the amplitude of the Payne effect decreases with increasing temperature which is opposite to the entropic variation characteristic of the unfilled network [23-25]. The decrease of the Payne effect results from a weakening of filler-filler interactions by raising the temperature.

\subsubsection{Tensile properties}

A common objective for adding fillers into polymers is to increase the modulus or stiffness. Composite theories have been developed in order to predict the performance of the composite by taking into account various filler parameters including geometry, stiffness and orientation [26-28]. In polymer-clay nanocomposites, it was demonstrated that the superior reinforcement provided by exfoliated layered aluminosilicates with regard to a conventional filler like glass fibers, arises primarily from the combination of high modulus and aspect ratio of the nanofiller [27].

As seen in Figure 6a which represents the strain dependence of the nominal stress for pure NR and for composites, considerable improvement in stiffness is observed upon addition of MWNTs in the NR matrix. In order to evaluate the extent of improvement with the nanotube loading, the stress at $100 \%$ strain is displayed in Figure 6b.

The stress-strain curve for unfilled NR exhibits a large increase in stress at higher deformations. NR

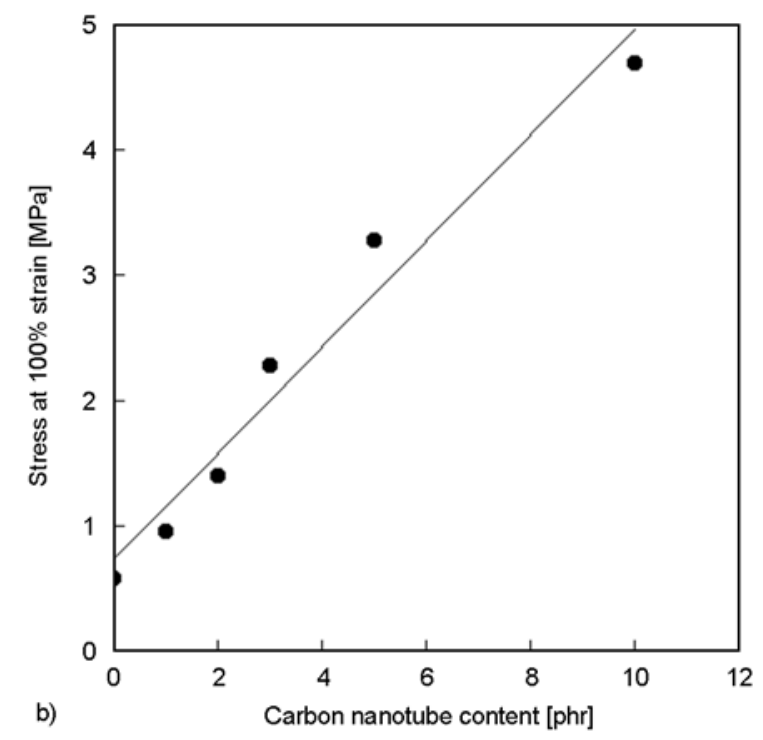

Figure 6. Stress-strain curves (a) and stress at 100\% for pure NR and MWNTs composites (b) 
displays, due to its uniform microstructure, a very unique important characteristic, that is, the ability to crystallize under strain, a phenomenon known as strain-induced crystallization. This phenomenon is responsible for the large and abrupt increase in the reduced stress observed at higher deformation corresponding, in fact, to a self-toughening of the elastomer because the crystallites act as additional cross-links in the network. This process can be better visualized by using a Mooney-Rivlin representation, based on the so-called Mooney-Rivlin equation (Equation (3)):

$$
\left[\sigma^{\star}\right]=\frac{\sigma}{\alpha-\alpha^{-2}}=2 C_{1}+2 C_{2} \alpha^{-1}
$$

where $\sigma$ is the nominal stress, $\alpha$ is the extension ratio and $2 C_{1}$ and $2 C_{2}$ are constants independent of $\alpha$. The curves of the unfilled vulcanizate displays, at higher deformations, an upturn in the modulus ascribed to the strain-induced crystallization of polymer chains (Figure 7). For the composites filled with 1 and $2 \mathrm{phr}$, the upturn is still observed but it starts at a lower extension ratio than that of the unfilled sample. That means that the addition of carbon nanotubes favors the crystallization process. This fact has already been established by infrared spectroscopy under strain [29] and by synchroton wide-angle X-ray diffraction [30]. The reduction in the strain at rupture for the other composites does not allow the observation of the tensile behaviour at high deformations. Unfortunately, the rupture prop-

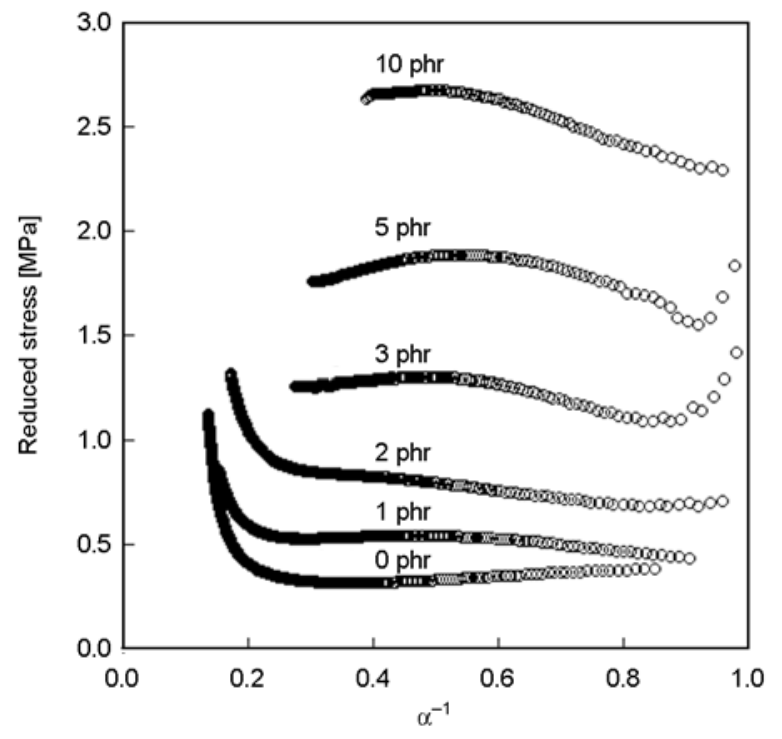

Figure 7. Mooney-Rivlin plots for pure NR and MWNTs/ NR composites erties are negatively affected by the nanotubes probably on account of the presence of some agglomerates which act as failure points and lead to a degradation of the mechanical properties of the materials. The stress at $100 \%$ deformation is seen to increase linearly with the MWNTs loading (Figure 6b). With regard to the pure polymer, $10 \mathrm{phr}$ of MWNTs leads to more than a $700 \%$ increase in the stress at $100 \%$ strain. The observed improvements are higher than those previously obtained in the literature for the same polymer [10, 29, 31-36].

Such increases in stiffness are not observed for similar loading fractions of spherical carbon black or silica particles in the same matrix, thus highlighting the effect of the high aspect ratio (length/diameter) of the nanotubes. In conventional composites, the increase in the modulus has been ascribed to a hydrodynamic effect arising from the inclusion of rigid particles in the soft matrix and to an increase in the cross-linking density created by polymerfiller interactions $[1,2,26,37-40]$. But the anisometry of the filler structures as well as the quality of their dispersion can greatly affect the composite performance. The latter point is especially true for entangled MWNTs in which the occluded rubber trapped inside the bundles and partially shielded from deformation, increases the effective filler concentration.

To interpret the variation of stiffness of the polymeric composites with the MWNTs amount, the $100 \%$ modulus results are fitted to the model of Halpin-Tsai [41]. intended to predict the mechanical properties of fibre reinforced composites. This model (Equation (4)) yields, for aligned fibre composites and in conditions where the modulus of the fiber, $E_{\mathrm{f}}$, is much higher than that of the unfilled matrix (as in elastomeric composites):

$$
E=E_{0} \frac{1+f \varphi}{1-\varphi}
$$

( $E$ and $E_{0}$ are the moduli of the composite and the unfilled elastomer respectively, $f$ is the aspect ratio and $\varphi$, the volume fraction of filler ).

The experimental values are compared with the Halpin-Tsai predictions using an aspect ratio of 90 to fit the data (Figure 8). This value is lower than expected from the average dimensions of the MWNTs but much higher than those previously pub- 


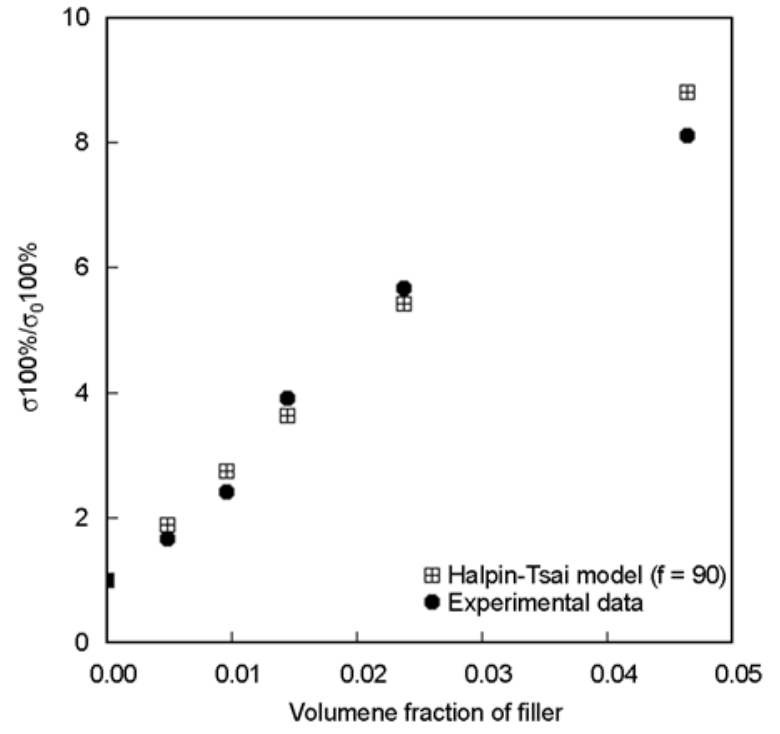

Figure 8. Dependence of the stress at $100 \%$ strain of NR/ MWNTs composites on the filler volume fraction and comparison with predictions of theoretical models

lished for hydrocarbon rubber/MWNTs composites $[42,43]$ as a result from a better filler dispersion.

Besides the state of dispersion, another fundamental issue that determines the properties of the composite is the interfacial interaction between the polymer and the nanotubes. A good adhesion between the two phases is required and might result in better load transfer from the matrix to the nanotube [44]. Raman spectroscopy under strain has also been used to probe interactions between carbon nanotubes and polymers in nanotube-based composites. If the nanotubes are carrying strain, the Raman peaks of carbon nanotubes have been shown to exhibit shifts upon application of a mechanical deformation to the composite. Large downshifts up to $20 \mathrm{~cm}^{-1}$ at about $1 \%$ strain have been observed for the $G^{\prime}$ band of SWNTs in stiff polymers such as epoxy resins [44-46]. In our rubbery composites, no significant shift of the $G^{\prime}$ band has been observed which means that load transfer to the nanotubes is negligible. However, in our experiments, we obtain an upshift in frequency of the $G$ mode (ascribed to the stretching of the $\mathrm{C}-\mathrm{C}$ bond of the carbon materials) with an increase in strain. The upshift of the $G$ mode has been attributed to nanotube-nanotube decoupling within the bundle because the upshift is not reversible with strain [47, 48].

Some hysteresis (area between the first and second stretches) is also observed in carbon nanotube-filled

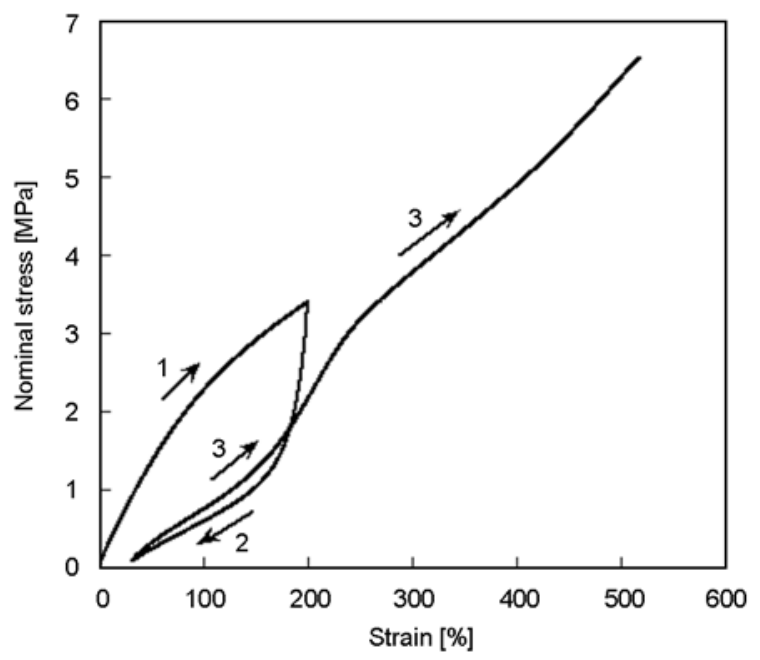

Figure 9. Loading-unloading cycles performed on NR filled with 3 phr of MWNTs

samples while the unfilled sample does not display any significant stress softening effect. In the experiment presented in Figure 9, the composite was stretched to a strain of $200 \%$, then released and restretched till the rupture of the sample. In view of our Raman results, we are in a position to believe that there is a poor adhesion between the nanotube surface and the rubber. Consequently, the stress-softening effect does not originate as in the case of conventional composites from a loss of elastic chains taking place at the polymer-filler interface [49]. In previous papers $[42,50]$, we have suggested a loss of nanotube orientation on releasing the strain to explain the lowering in the stress in the second stretching. Despite the fact that we cannot overlook the large contribution of orientational aspects in the stress-strain behavior of composites, we can also consider that a significant effect of the strain is a debundling of the agglomerates still remaining in the polymeric medium. This interpretation is supported by the fact that the rupture of the sample in the second stretching occurs at a much higher value of strain than that obtained in the first stretching curve (compare Figure 6a and 9).

\section{Conclusions}

This paper demonstrates that optimized processing conditions used to achieve good dispersion lead to composites with high mechanical and electrical properties. Nevertheless, the presence of a small number of agglomerates acting as defects are responsible of the lack of improvement in rupture properties. Comparison of the experimental tensile meas- 
urements with the Halpin-Tsai model leads an aspect ratio of 90 for carbon nanotubes. This high aspect ratio appears to be the main parameter for mechanical reinforcement and electrical conduction. The high aspect ratio also explains the formation of a filler network at a low nanotube loading. Application of an uniaxial deformation to the composite leads to an increase of electrical resistivity while a drop in the storage modulus is observed when the sample is submitted to low shear strains. Both effects are attributed to the breakdown of the filler network.

\section{Acknowledgements}

This work has been carried out with the help of the 'Prix de la Recherche Jean Langlois 2009'. The author thanks Gilles Garnaud, Bettina Subtil and Sandra Hilaire for technical assistance.

\section{References}

[1] Bokobza L.: Elastomeric composites. I. Silicone composites. Journal of Applied Polymer Science, 93, 20952104 (2004).

DOI: 10.1002/app.20684

[2] Bokobza L.: The reinforcement of elastomeric networks by fillers. Macromolecular Materials and Engineering, 289, 607-621 (2004).

DOI: $10.1002 /$ mame.200400034

[3] Khare R., Bose S.: Carbon nanotube based composites - A review. Journal of Minerals and Materials Characterization and Engineering, 4, 31-46 (2005).

[4] Hu Y., Shenderova O. A., Hu Z., Padgett C. W., Brenner D. W.: Carbon nanostructures for advanced composites. Reports on Progress in Physics, 69, 18471895 (2006).

DOI: $10.1088 / 0034-4885 / 69 / 6 / R 05$

[5] Spitalsky Z., Tasis D., Papagelis K., Galiotis C.: Carbon nanotube-polymer composites: Chemistry, processing, mechanical and electrical properties. Progress in Polymer Science, 35, 357-401 (2010).

DOI: 10.1016/j.progpolymsci.2009.09.003

[6] Thongruang W., Balik C. M., Spontak R. J.: Volumeexclusion effects in polyethylene blends filled with carbon black, graphite, or carbon fiber. Journal of Polymer Science Part B: Polymer Physics, 40, 1013-1025 (2002).

DOI: 10.1002/polb.10157

[7] Bokobza L., Rahmani M., Belin C., Bruneel J-L., El Bounia N-E.: Blends of carbon blacks and multiwall carbon nanotubes as reinforcing fillers for hydrocarbon rubbers. Journal of Polymer Science Part B: Polymer Physics, 46, 1939-1951 (2008).

DOI: $10.1002 /$ polb.21529
[8] Ma P-C., Liu M-Y., Zhang H., Wang S-Q., Wang R., Wang K., Wong Y-K., Tang B-Z., Hong S-H., Paik KW., Kim J-K.: Enhanced electrical conductivity of nanocomposites containing hybrid fillers of carbon nanotubes and carbon black. ACS Applied Materials and Interfaces, 1, 1090-1096 (2009).

DOI: $10.1021 / \mathrm{am} 9000503$

[9] Mamunya Ye. P., Davydenko V. V., Pissis P., Lebedev E. V.: Electrical and thermal conductivity of polymers filled with metal powders. European Polymer Journal, 38, 1887-1897 (2002) DOI: $10.1016 / \mathrm{S} 0014-3057(02) 00064-2$

[10] Bokobza L., Kolodziej M.: On the use of carbon nanotubes as reinforcing fillers for elastomeric materials. Polymer International, 55, 1090-1098 (2006).

DOI: $10.1002 /$ pi.2064

[11] Das N. C., Chaki T. K., Khastgir D.: Effect of axial stretching on electrical resistivity of short carbon fibre and carbon black filled conductive rubber composites. Polymer International, 51, 156-163 (2002).

DOI: $10.1002 /$ pi.811

[12] Yamaguchi K., Busfield J. J. C., Thomas A. G.: Electrical and mechanical behavior of filled elastomers. I. The effect of strain. Journal of Polymer Science Part B: Polymer Physics, 41, 2079-2089 (2003).

DOI: $10.1002 /$ polb.10571

[13] Busfield J. J. C., Thomas A. G., Yamaguchi K.: Electrical and mechanical behavior of filled elastomers 2 : The effect of swelling and temperature. Journal of Polymer Science Part B: Polymer Physics, 42, 21612167 (2004).

DOI: $10.1002 /$ polb.20085

[14] Kang J. H., Park C., Scholl J. A., Brazin A. H., Holloway N. M., High J. W., Lowther S. E., Harrison J. S.: Piezoresistive characteristics of single wall carbon nanotube/polyimide nanocomposites. Journal of Polymer Science Part B: Polymer Physics, 47, 994-1003 (2009).

DOI: $10.1002 /$ polb.21705

[15] Kujawski M., Pearse J. D., Smela E.: Elastomers filled with exfoliated graphite as compliant electrodes. Carbon, 48, 2409-2417 (2010). DOI: $10.1016 /$ j.carbon.2010.02.040

[16] Ciselli P., Lu L., Busfield J. J. C., Peijs T.: Piezoresistive polymer composites based on EPDM and MWNTs for strain sensing applications. e-Polymers, no.014 (2010).

[17] Bokobza L.: New developments in rubber reinforcement. Kautschuk Gummi Kunststoffe, 62, 23-27 (2009).

[18] Bokobza L.: Enhanced electrical and mechanical properties of multiwall carbon nanotube rubber composites. Polymers for Advanced Technologies, submitted (2011).

[19] Wang M-J.: Effect of polymer-filler and filler-filler interactions on dynamic properties of filled vulcanizates. Rubber Chemistry and Technology, 71, 520589 (1998).

DOI: $10.5254 / 1.3538492$ 
[20] Clément F., Bokobza L., Monnerie L.: Investigation of the Payne effect and its temperature dependence on silica-filled polydimethylsiloxane networks. Part I: Experimental results. Rubber Chemistry and Technology, 78, 211-231 (2005).

DOI: $10.5254 / 1.3547879$

[21] Clément F., Bokobza L., Monnerie L.: Investigation of the Payne effect and its temperature dependence on silica-filled polydimethylsiloxane networks. Part II: Test of quantitative models. Rubber Chemistry and Technology, 78, 232-244 (2005).

DOI: $10.5254 / 1.3547880$

[22] Ramier J., Gauthier C., Chazeau L., Stelandre L., Guy L.: Payne effect in silica-filled styrene-butadiene rubber: Influence of surface treatment. Journal of Polymer Science Part B: Polymer Physics, 45, 286-298 (2007). DOI: $10.1002 /$ polb.21033

[23] Payne A. R.: The dynamic properties of carbon blackloaded natural rubber vulcanizates. Part I. Journal of Applied Polymer Science, 6, 57-63 (1962).

DOI: 10.1002/app.1962.070061906

[24] Payne A. R., Whittaker R. E.: Low strain dynamic properties of filled rubbers. Rubber Chemistry and Technology, 44, 440-478 (1971). DOI: $10.5254 / 1.3547375$

[25] Maier P. G., Göritz D.: Molecular interpretation of the Payne effect. Kautschuk Gummi Kunststoffe, 49, 1824 (1996).

[26] Ahmed S., Jones F. R.: A review of particulate reinforcement theories for polymer composites. Journal of Materials Science, 25, 4933-4942 (1990).

DOI: $10.1007 / \mathrm{BF} 00580110$

[27] Fornes T. D., Paul D. R.: Modeling properties of nylon 6/clay nanocomposites using composite theories. Polymer, 44, 4993-5013 (2003).

DOI: 10.1016/S0032-3861(03)00471-3

[28] Wu Y-P., Jia Q-X., Yu D-S., Zhang L-Q.: Modeling Young's modulus of rubber-clay nanocomposites using composite theories. Polymer Testing, 23, 903909 (2004) DOI: 10.1016/j.polymertesting.2004.05.004

[29] Kolodziej M., Bokobza L., Bruneel J-L.: Investigations on natural rubber filled with multiwall carbon nanotubes. Composite Interfaces, 14, 215-228 (2007). DOI: $10.1163 / 156855407780340304$

[30] Weng G., Huang G., Qu L., Nie Y., Wu J.: Large-scale orientation in a vulcanized stretched natural rubber network: Proved by in situ synchrotron X-ray diffraction characterization. The Journal of Physical Chemistry $\mathrm{B}, \mathbf{1 1 4}, 7179-7188$ (2010).

DOI: $10.1021 / \mathrm{jp} 100920 \mathrm{~g}$

[31] Fakhru'l-Razi A., Atieh M. A., Girun N., Chuah T. G., El-Sadig M., Biak D. R. A.: Effect of multi-wall carbon nanotubes on the mechanical properties of natural rubber. Composite Structures, 75, 496-500 (2006). DOI: $\underline{10.1016 / \mathrm{j} . c o m p s t r u c t .2006 .04 .035}$
[32] Bhattacharyya S., Sinturel C., Bahloul O., Saboungi M-L., Thomas S., Salvetat J-P.: Improving reinforcement of natural rubber by networking of activated carbon nanotubes. Carbon, 46, 1037-1045 (2008).

DOI: $10.1016 /$ j.carbon.2008.03.011

[33] Cataldo F., Ursini O., Angelini G.: MWCNTs elastomer nanocomposite, Part 2: The addition of MWCNTs to an oil-extended SBR-based carbon black-filled rubber compound. Fullerenes, Nanotubes and Carbon Nanostructures, 17, 55-66 (2009).

DOI: $10.1080 / 15363830802515923$

[34] Nah C., Lim J. Y., Cho B. H., Hong C. K., Gent A. N.: Reinforcing rubber with carbon nanotubes. Journal of Applied Polymer Science, 118, 1574-1581 (2010). DOI: 10.1002/app.32524

[35] Sui G., Zhong W. H., Yang X. P., Yu Y. H.: Curing kinetics and mechanical behavior of natural rubber reinforced with pretreated carbon nanotubes. Materials Science and Engineering: A, 485, 524-531 (2008). DOI: $10.1016 /$ j.msea.2007.09.007

[36] Ismail H., Ramly F., Othman N.: Multiwall carbon nanotube-filled natural rubber: The effects of filler loading and mixing method. Polymer-Plastics Technology and Engineering, 49, 260-266 (2010). DOI: $10.1080 / 03602550903413888$

[37] Dannenberg E. M.: The effects of surface chemical interactions on the properties of filler-reinforced rubbers. Rubber Chemistry and Technology, 48, 410-444 (1975).

DOI: $10.5254 / 1.3547460$

[38] Wagner M. P.: Reinforcing silicas and silicates. Rubber Chemistry and Technology, 49, 703-774 (1976). DOI: $10.5254 / 1.3534979$

[39] Voet A.: Reinforcement of elastomers by fillers: Review of period 1967-1976. Journal of Polymer Science: Macromolecular Reviews, 15, 327-373 (1980). DOI: $10.1002 /$ pol.1980.230150107

[40] Edwards D. C.: Polymer-filler interactions in rubber reinforcement. Journal of Materials Science, 25, 41754185 (1990). DOI: $10.1007 / \mathrm{BF} 00581070$

[41] Halpin J. C.: Stiffness and expansion estimates for oriented short fiber composites. Journal of Composite Materials, 3, 732-734 (1969). DOI: $10.1177 / 002199836900300419$

[42] Bokobza L.: Multiwall carbon nanotube elastomeric composites: A review. Polymer, 48, 4907-4920 (2007). DOI: $10.1016 /$ j.polymer.2007.06.046

[43] Perez L. D., Zuluaga M. A., Kyu T., Mark J. E., Lopez B. L.: Preparation, characterization, and physical properties of multiwall carbon nanotube/elastomer composites. Polymer Engineering and Science, 49, 866874 (2009).

DOI: $10.1002 /$ pen.21247 
[44] Frogley M. D., Ravich D., Wagner H. D.: Mechanical properties of carbon nanoparticle-reinforced elastomers. Composites Science and Technology, 63, 16471654 (2003).

DOI: $10.1016 / \mathrm{S} 0266-3538(03) 00066-6$

[45] Cooper C. A., Young R. J., Halsall M.: Investigation into the deformation of carbon nanotubes and their composites through the use of Raman spectroscopy. Composites Part A: Applied Science and Manufacturing, 32, 401-411 (2001).

DOI: 10.1016/S1359-835X(00)00107-X

[46] Kao C. C., Young R. J.: A Raman spectroscopic investigation of heating effects and the deformation behaviour of epoxy/SWNT composites. Composites Science and Technology, 64, 2291-2295 (2004). DOI: $10.1016 /$ j.compscitech.2004.01.019
[47] Bokobza L.: Vibrational spectroscopic and mechanical investigation of carbon nanotube-reinforced styrenebutadiene rubbers. Macromolecular Symposia, 305, 19 (2011)

DOI: 10.1002/masy.201000119

[48] Bokobza L.: A Raman investigation of carbon nanotubes embedded in a soft polymeric matrix. Journal of Inorganic and Organometallic Polymers and Materials, in press (2011).

DOI: $10.1007 / \mathrm{s} 10904-011-9590-7$

[49] Clément F., Bokobza L., Monnerie L.: On the Mullins effect in silica-filled polydimethylsiloxane networks. Rubber Chemistry and Technology, 74, 847-870 (2001). DOI: $10.5254 / 1.3547657$

[50] Bokobza L., Belin C.: Effect of strain on the properties of a styrene-butadiene rubber filled with multiwall carbon nanotubes. Journal of Applied Polymer Science, 105, 2054-2061 (2007).

DOI: $10.1002 /$ app. 26153 